\title{
Looking into the evolution of granular asteroids in the Solar System
}

\author{
Paul Sánchez ${ }^{1, \star}$, Daniel Scheeres ${ }^{2, \star \star}$, Masatoshi Hirabayashi ${ }^{3, \star \star \star}$, and Simon Tardivel ${ }^{2, \star \star \star \star}$ \\ ${ }^{1}$ Colorado Center for Astrodynamics Research, University of Colorado Boulder, 431 UCB, Boulder, CO 80309-0431, USA \\ ${ }^{2}$ Aerospace Engineering Sciences, University of Colorado Boulder, 429 UCB, Boulder, CO 80309-0429, USA \\ ${ }^{3}$ Department of Earth and Atmospheric Sciences, Purdue University, 550, West Lafayette, IN 47907, USA
}

\begin{abstract}
By now it has been accepted that most of the small asteroids in the Solar System are granular aggregates kept together by gravitational and possibly, cohesive forces. These aggregates can form, deform and disrupt over millennia subjected to different internal and external factors that would ultimately determine how they evolve over time. Parameters such as porosity, cohesive and tensile strength, angles of friction, particle size distributions, stress states, heterogeneity and yield criteria among others, determine how these granular systems will react when subjected to different, changing, external factors. These external factors include solar photon momentum, gravitational tides, micro- and macro-impacts and are believed to have produced and shaped the current asteroid population. In our research we use a combination of Soil Mechanics theory, Soft-Sphere Discrete Element Method (SSDEM) Simulations and Orbital Mechanics in order to understand how simulated, homogeneous and heterogeneous, ellipsoidal and spherical gravitational aggregates, a crude but useful representation of an asteroid, evolve when rotated to the point of disruption. Then, we compare our results to the shapes of observed asteroids as well as to the disruption patterns of a few active asteroids. Our results lead us to believe that the different shapes of observed asteroids as well as their unique disruption patterns could give us clues about their internal structure, strength and geophysical properties in general.
\end{abstract}

\section{Granular Asteroids and YORP}

In the last decade, the study of asteroids as granular aggregates has gained importance. Even though it was originally assumed that the smallest of asteroids were monolithic rocks with a bare surface, the pictures obtained by NASA Galileo, Near-Showmaker (or just NEAR) and JAXA Hayabusa space missions revealed a substantial layer of unconsolidated rocky material and dust (regolith) covering the surface of (951) Gaspra, (243) Ida, (433) Eros $[1,2]$ and (25143) Itokawa. Additionally, observations made by the Hubble Space Telescope of the disruption events of active asteroids P2013/R3 [3] and P2013/P5 [4] imply that not only asteroid surfaces are covered by regolith, but that their internal structure is not monolithic. This is what we define as a granular asteroid, a naturally occurring self-gravitating granular system.

Of the many events that can disrupt a granular, nonmonolithic asteroid, here we will focus only on the disruption that could be caused by rotation. The angular velocity of this rotation, in the context of NEOs, can be accelerated by the Yarkovsky-O'Keefe- Radzievskii-Paddack (YORP) effect which results from an interplay between solar radiation pressure and the shape of an asteroid [5,6]. Very succinctly, when the photons that come from the Sun im-

\footnotetext{
^e-mail: diego.sanchez-lana@colorado.edu

$\star \star$ e-mail: dscheeres@colorado.edu

$\star \star \star$ e-mail: thirabayashi@purdue.edu

$\star \star \star \star$ e-mail: simon.tardivel@ colorado.edu
}

pact on the surface of an asteroid, they will be reflected, absorbed and re-emitted from it anisotropically due to its irregular shape. This will produce a net torque that affects the angular velocity of the asteroid. Over millennia, this change in the angular velocity will produce the deformation or disruption of the asteroid [7]. A clear signature of the YORP effect was first observed in asteroid 54509 YORP [5, 8] and (1862) Apollo [9].

If the strength of granular asteroids were governed by gravitational forces alone, a plot of their size (or absolute magnitude $\mathrm{H}$, more appropriately) versus spin rate would show a very clear limit for the latter, which could give us a way to estimate the bulk density of these bodies if an albedo (proportion of reflected sunlight) of 0.2 is assumed. This is exactly what happens for most asteroids, but not for all. The observed spin barrier shows that larger asteroids have a limiting rotation period of $\approx 2.2 \mathrm{~h}$ [10]. Small asteroids $(\mathrm{H}<21.5 \mathrm{mag})$ with sizes $<150 \mathrm{~m}$ can rotate much faster as was first described by [10] and reconfirmed by later surveys $[11,12]$. This is what has led scientists to believe that cohesive forces are important for these granular systems [13-16].

\section{Cohesive Forces}

However, if we say that cohesive forces are important, we need to understand where they come from and what their effects are, if any at all, for the evolution of asteroids. If we 

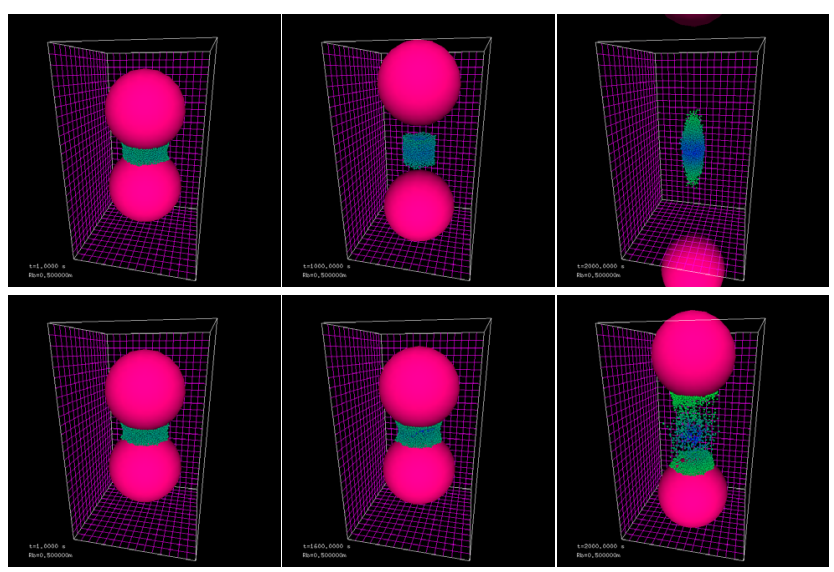

Figure 1. SSDEM simulations. Time evolution of two selfgravitating granular bridge: (top) cohesionless and (bottom) cohesive. The latter needed a pulling force about 40 times greater than the one on top to fail catastrophically [17].

suppose that asteroids are free of volatiles that could bond their constituent particles and provide the tensile strength needed, we are left with another option and this is van der Waals forces. In a terrestrial environment, these forces are mostly negligible, except for powders as the weight of each particle is comparable to their cohesive attractive force. In the asteroid environment however, where ambient gravity is in the order of milli- to micro-g, cohesion and weight become comparable for larger, centimeter size, particles [15]. An asteroid, of course, is not made up only of pebbles, but it has particles that range from microns to tens of meters. A system as such is impossible to simulate with the available computational resources. However, simpler systems, that show the effect of cohesive forces on a self-gravitating granular system, can be constructed and studied. Fig. 1 shows one such systems.

Fig. 1 shows a granular equivalent to a liquid bridge. In these self-gravitating systems, we have two $1 \mathrm{~m}$ boulders, joined together by a bridge formed by thousands of centimeter size particles. The system on top is cohesionless, whereas the one at the bottom is cohesive. The boulders are pulled in opposite directions by a force increasingly stronger than their mutual gravitational attraction to measure the tensile strength of the bridge. This study [17] showed that the tensile strength of a cohesive aggregate is inversely proportional to the average particle size of the particles forming it (porosity, Hamaker constant, coordination number and others are considered constants). Basically, the smaller dust and regolith in an asteroid could be seen as a weak cement, a matrix in which the larger boulders are embedded. This effect is as important as gravity for small asteroids, but being independent of mass, it looses its preponderance for larger asteroids where gravitational effects dominate. This study also showed that it should be possible to simulate real size asteroids with much less particles if we simply add a particle-particle cohesive force that depends on a predetermined average particle size. This being so, we could now be in a po- sition to carry out realistic simulations of asteroids with levels of tensile strength that are related to their physical properties (particle size, porosity, etc.). This would help us understand the effects of different levels of strength on self-gravitating cohesive aggregates that are spun up to deformation/disruption levels.

\section{Disruption Patterns}

Exact solutions for the deformation of self-gravitating aggregates have been obtained for spherical and ellipsoidal shapes, but these solutions could not predict how a disruption process would occur. To solve this problem Sánchez and Scheeres[18] carried out a survey on angle of friction and tensile strength for ellipsoidal and spherical selfgravitating aggregates which were quasi-statically spun up to disruption. Fig. 2 shows some of the produced disruption patterns.
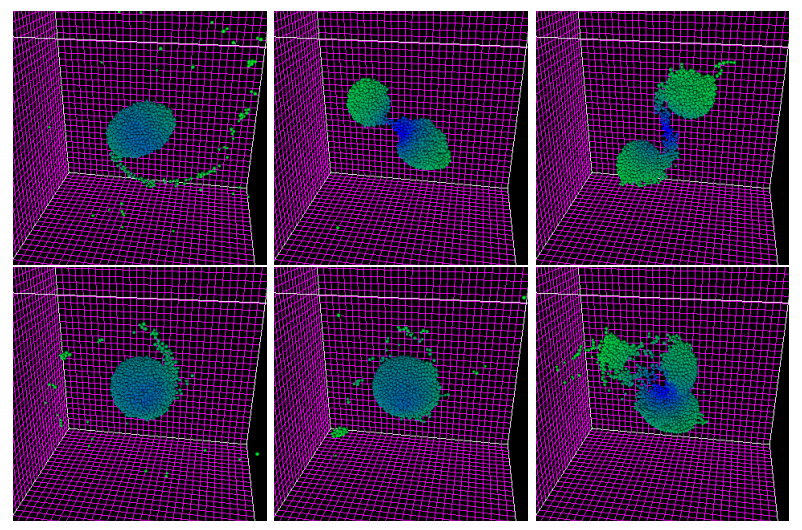

Figure 2. Different disruption patterns for ellipsoidal (top) and spherical (bottom) aggregates (angle of friction $\approx 35^{\circ}$ ) with different tensile strengths $\left(\sigma_{y y}^{a}\right)$. From left to right: $0 \mathrm{~Pa}, 1.63 \mathrm{~Pa}$ and $16.05 \mathrm{~Pa}$. Size $\approx 142 \mathrm{~m} \mathrm{[18].}$

This study revealed that if the angle of friction of an aggregate defined how much it would deform, tensile strength would determine how it would lose mass. This is, low or no tensile strength would result in a particleby-particle detachment and high tensile strength, in big chunks being ejected. For an angle of friction of $\approx 35^{\circ}$ and at high enough values, the aggregate would be catastrophically disrupted. Incidentally, this could also provide a possible mechanism for the formation of binary asteroids and asteroid pairs, though this is still to be corroborated. In spite of this, the characteristic shapes of asteroids (1999 KW4, 1950 DA and Geographos) could be observed in the simulated aggregates, though the latter were much more deformed. Deformation could be diminished by strengthening the aggregates, but this would result in the ejected piece being to large to form a satellite. The solution to this dilemma could come in the form of aggregates with a core that is stronger than the outer shell; this is discussed next.

\section{The Core of a Gravitational Aggregate}

Most studies about small asteroids have made the common assumption that their structure was homogeneous. How- 
ever, there is no reason to believe that this is so, especially if we observe the heterogeneity of their surfaces [19, 20]. Additionally, previous studies carried out by Hirabayahi and Scheeres [21, 22] had shown that aggregates with denser, stronger cores would strengthen the whole structure and reproduce surface slopes similar to those found in $1999 \mathrm{KW} 4$. It was also shown that for such a bicone shape, a homogeneous structure would first fail at its centre and not at the surface which, in the case of asteroid 1950 DA, would result in a weak interior due to shearing [23]. On the other hand, the results of having a core and shell with different cohesive strengths were yet to be studied.

For this study [24], the same simulation setup shown in fig. 2 was used, with the difference that the tensile strength of the shell was 5 times weaker than its core. Only spherical shapes were used and the aggregates were spun up to disruption rates. The size of the core was changed in order to observed the variations in shape. From this study it was concluded that the failure conditions derived by the analytical model were consistent with those by the SSDEM [24]. The results showed that an aggregate with homogeneous cohesion would always fail internally before it fails superficially. To have surface shedding, therefore, the progenitor body needed a strong core so that only the surface region failed structurally. The simulations also exposed the dynamics of the initial failure phase. The primary result was that for all the cases, a granular spheroid would fail locally through a severe material flow on its surface. This came from the fact that since the initial body configuration was not perfectly uniform, which we believe is more realistic, some surface regions were more susceptible to failure than other regions. Interestingly enough, an aggregate with a core with a radius of $0.7 R_{b}\left(R_{b}\right.$ is the radius of the aggregate) would produce slopes that match asteroid 1999 KW4 almost perfectly [25]. For an aggregate with a core of radius $0.9 R_{b}$, deformation would be minimal and the ejected matter would leave a hole close to the equator.

Aggregates with weak cores are also interesting to study as this should also be possible. We again start from the same configuration as before, weaken the core with respect to the shell and spin up the aggregate to induce its disruption. As expected, the aggregates fail structurally earlier than their homogeneous counterparts, but for a core of $0.5 R_{b}$ something interesting happens. The shape into which the aggregate evolves in strikingly similar to that of asteroid Itokawa; this can be observed in fig. 3. This simulation shows that the deformation of the body causes the subsurface material of the shell to be exposed (magenta particles). This exposed material is located on the larger lobe of the body and towards the neck and also in the back of the body. These are the exact places where asteroid Itokawa shows areas with smaller regolith.

If we apply all these results to the recent observations of active asteroids P/2013 R3 and P/2013 P5, we could conclude that the former needed a strong core to form the observed tails and that the latter had at best a homogeneous interior. Asteroid 1999 KW4 could have a strong core, and asteroid Itokawa could possibly have a weak interior [6]. As encouraging as all these results and comparisons
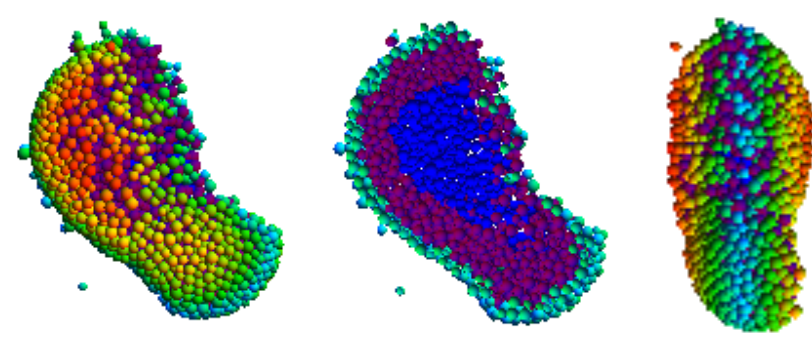

Figure 3. Final stage of an initially spherical aggregate with a $0.5 R_{b}$ weak core. The image at the left and right show the view from the side and bottom respectively. The centre image is a cross-sectional cut. The blue particles form the core, the magenta particles form the inner part of the shell.

are, we still have not observed evidence that the formation mechanism here proposed has actually happened. However, there is possibly a piece of evidence that might have been mistaken for a crater when it could be in fact evidence of fission via rotation.

\section{The curious case of 2008 EV5}

Asteroid 2008 EV5 is $450 \mathrm{~m}$ in diametre and has a symmetrical, wide bicone shape with one peculiarity, it has a $20 \mathrm{~m}$ deep cavity on its equatorial ridge. It has been previously explained that this feature could have been formed by an impact [26]. However, in the best case scenario, the probability of having an impact at that precise location and in the required time frame, taking into account the YORP effect, is extremely low $(<0.01 \%)$. Though not impossible, it is highly improbable and so we propose a different explanation.

As the shape model of this asteroid is known to great resolution, it is possible to compute its gravity field with fine accuracy. As angular velocity is increased, the effective force felt by a particle at its surface, sum of the gravity acceleration and centrifugal acceleration, becomes increasingly tenuous. Eventually, for an asymmetric asteroid, some regions see this balance reverse, and they feel an outward pull. The key observation we made here is that these pulled-out regions were limited to a very specific and identified portions of the asteroid.

We then make the assumption that the cavity in 2008 EV5 was initially filled with material (see fig. 4). In that case, the filled cavity is precisely the portion of the asteroid that feels this outward pull at high spin rate. We calculated that such a piece would require under $1 \mathrm{~Pa}$ of tensile strength to be fissioned, leaving the observed cavity behind. This begs another question, how could the equatorial region have such a low tensile strength when in other asteroids this has been measured at values between 50-250 Pa?

We have already explained that at high enough spin rates, aggregates with a strong core would produce material flow towards the equatorial region. This flow should be conducive to particle size segregation [27], leaving the larger ones to form the equatorial ridge. This flow would produce a region formed by larger particles in which 


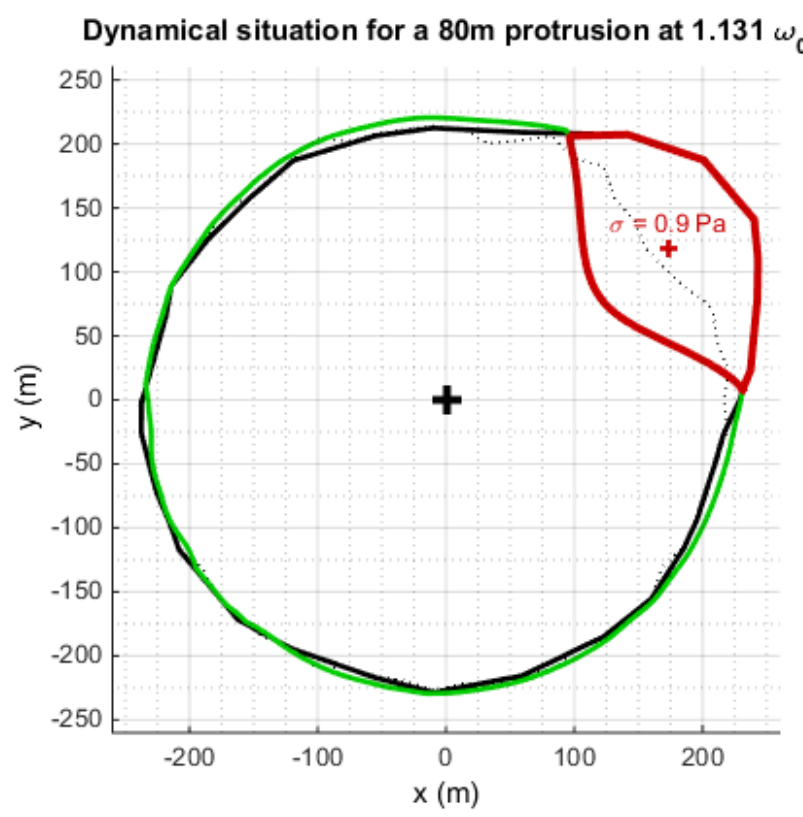

Figure 4. Planar cut of asteroid 2008 EV5. The ridge line is in green. The red line represents the region feeling an outward pull. The black line represents the altered asteroid contour, and the dotted line is the actual asteroid shape.

porosity as well as the average particle size have increased, allowing for a lower tensile strength. A similar argument could be done for asteroid 2000 DP107 [28].

\section{Conclusions}

In this paper we have presented a study of the effects of cohesive forces and heterogeneities in self-gravitating aggregates and their application to small asteroids in the Solar System. We have shown how van der Waals forces between the grains that form an asteroid, and other bulk properties, are very important to determine the tensile strength of these aggregates. In turn, its specific value in comparison to gravitational forces could determine specific disruption patterns at elevated spin rates. If we now take into account that asteroids can be heterogeneous, our study suggests that the current asteroid shapes and disruption patterns should reflect their internal structure.

\section{References}

[1] R.J. Sullivan, P.C. Thomas, S.L. Murchie, M.S. Robinson, Asteroids III pp. 331-350 (2002)

[2] M.S. Robinson, P.C. Thomas, J. Veverka, S.L. Murchie, B.B. Wilcox, Meteoritics and Planetary Science 37, 1651 (2002)

[3] D. Jewitt, J. Agarwal, J. Li, H. Weaver, M. Mutchler, S. Larson, The Astrophysical Journal Letters 784, L8 (2014)

[4] D. Jewitt, J. Agarwal, H. Weaver, M. Mutchler, S. Larson, The Astrophysical Journal Letters 778, L21 (2013)
[5] P.A. Taylor, J.L. Margot, D. Vokrouhlický, D.J. Scheeres, P. Pravec, S.C. Lowry, A. Fitzsimmons, M.C. Nolan, S.J. Ostro, L.A.M. Benner et al., Science 316, 274 (2007)

[6] S. Lowry, P. Weissman, S. Duddy, B. Rozitis, A. Fitzsimmons, S. Green, M. Hicks, C. Snodgrass, S. Wolters, S. Chesley et al., Astronomy and Astrophysics 562, 48 (2014)

[7] D.P. Rubincam, Icarus 148, 2 (2000)

[8] S.C. Lowry, A. Fitzsimmons, P. Pravec, D. Vokrouhlický, H. Boehnhardt, P.A. Taylor, J.L. Margot, A. Galád, M. Irwin, J. Irwin et al., Science 316, 272 (2007)

[9] M. Kaasalainen, J. Ďurech, B. Warner, Y. Krugly, N. Gaftonyuk, Nature 446, 420 (2007)

[10] P. Pravec, A.W. Harris, Icarus 148, 12 (2000)

[11] B.D. Warner, A.W. Harris, P. Pravec, Icarus 202, 134 (2009)

[12] P.A. Taylor, E.S. Howell, M.C. Nolan, A.A. Thane, The Shape and Spin Distributions of Near-Earth Asteroids Observed with the Arecibo Radar System, in American Astronomical Society Meeting Abstracts \#220 (2012), Vol. 220 of American Astronomical Society Meeting Abstracts, p. 128.02

[13] K.A. Holsapple, Icarus 172, 272 (2004)

[14] K.A. Holsapple, Icarus 205, 430 (2010)

[15] D. Scheeres, C. Hartzell, P. Sánchez, M. Swift, Icarus 210, 968 (2010)

[16] D.P. Sánchez, D.J. Scheeres, Icarus 218, 876 (2012)

[17] P. Sánchez, D.J. Scheeres, Meteoritics \& Planetary Science 49, 788 (2014)

[18] P. Sánchez, D.J. Scheeres, Icarus 271, 453 (2016)

[19] A. Fujiwara, J. Kawaguchi, D.K. Yeomans, M. Abe, T. Mukai, T. Okada, J. Saito, H. Yano, M. Yoshikawa, D.J. Scheeres et al., Science 312, 1330 (2006)

[20] H. Yano, T. Kubota, H. Miyamoto, T. Okada, D. Scheeres, Y. Takagi, K. Yoshida, M. Abe, S. Abe, O. Barnouin-Jha et al., Science 312, 1350 (2006)

[21] M. Hirabayashi, Icarus 236, 178 (2014)

[22] D. Scheeres, Icarus 247, 1 (2015)

[23] M. Hirabayashi, D.J. Scheeres, The Astrophysical Journal Letters 798, L8 (2015)

[24] M. Hirabayashi, D.P. Sánchez, D.J. Scheeres, The Astrophysical Journal 808, 63 (2015)

[25] P. Sánchez, Proceedings of the International Astronomical Union 10, 111 (2015)

[26] M.W. Busch, S.J. Ostro, L.A.M. Benner, M. Brozovic, J.D. Giorgini, J.S. Jao, D.J. Scheeres, C. Magri, M.C. Nolan, E.S. Howell et al., Icarus 212, 649 (2011), 1101. 3794

[27] N. Thomas, Phys. Rev. E 62, 961 (2000)

[28] S.P. Naidu, J.L. Margot, P.A. Taylor, M.C. Nolan, M.W. Busch, L.A.M. Benner, M. Brozovic, J.D. Giorgini, J.S. Jao, C. Magri, The Astronomical Journal 150, 54 (2015) 